\title{
Are Suicide Attempters Wired Differently?
}

\section{A Comparison With Nonsuicidal Depressed Individuals Using Plan Analysis}

\author{
Juliane Brüdern, MSc, * Thomas Berger, PhD, * Konrad Michel, PhD, $\dagger$ Anja Gysin Maillart, PhD, $\dagger$ \\ Isabelle Schmutz Held, PhD, * and Franz Caspar, PhD*
}

\begin{abstract}
Limited research exists on internal risk processes in suicide attempters and factors that distinguish them from nonsuicidal depressive individuals. In this qualitative study, we investigated Plans, motives, and underlying self-regulatory processes of the two groups and conducted a comparative analysis. We analyzed narrative interviews of 17 suicide attempters and intake interviews of 17 nonsuicidal depressive patients using Plan Analysis. Then, we developed a prototypical Plan structure for both groups. Suicidal behavior serves various Plans found only in suicide attempters. Plans of this group are especially related to social perfectionism and withdrawal to protect their self-esteem. Depressive patients use several interpersonal control and coping strategies, which might help prevent suicidal behavior. The prototypical Plan structure of suicide attempters may be a valuable tool for clinicians to detect critical Plans and motives in their interaction with patients, which are related to suicide risk.
\end{abstract}

Key Words: Suicide, Depression, Risk processes, Self-regulation

(J Nerv Ment Dis 2015;203: 514-521)

Ev very year, approximately 1 million people worldwide commit suicide and between 10 million and 20 million attempt suicide (World Health Organization, 2008). Suicide is among the ten leading causes of death, and it is estimated that in 2020, suicide will represent $2.4 \%$ of the global burden of disease (World Health Organization, 2008). In the past two decades, a considerable number of models trying to understand suicidal behavior have been developed. The main focus has been identifying risk factors of suicidal behavior and associated high-risk groups. For example, mood disorders are a frequently discussed risk factor for suicidal behavior. Bertolote and Fleischmann (2002) found that in the general population, $35.8 \%$ of individuals who committed suicide had a mood disorder. The highest risk of committing suicide is during a major depressive episode; $3.2 \%$ of individuals who experience a major depression die of suicide (Blair-West et al., 1999). After a closer examination, it seems that depression predicts suicidal ideation, but not suicide plans or attempts (Nock et al., 2010). Powell et al. (2000) found in a study with psychiatric inpatients that on the basis of identified risk factors (e.g., chronic mental illness, family history of suicide), only two of the patients who committed suicide had a predicted risk of suicide above $5 \%$. These findings imply that suicidal behavior is more than a psychiatric diagnosis, such as depression, and should be approached as an independent behavior that must be examined separately (Linehan, 2008). Hence, the understanding of proximal processes that lead to suicidal behavior is crucial and can be clinically useful. Until now, research surrounding the internal risk processes of suicidal behavior has been limited. Furthermore, we know little about the mechanisms that may distinguish suicide attempters from depressive individuals who do not

*University of Bern; and †University Hospital of Psychiatry, Bern, Switzerland. Send reprint requests to Juliane Brüdern, MSc, Department of Clinical Psychology and Psychotherapy, University of Bern, Fabrikstrasse 8, 3012 Bern, Switzerland. E-mail: juliane.bruedern@psy.unibe.ch.

Copyright (C) 2015 Wolters Kluwer Health, Inc. All rights reserved.

ISSN: 0022-3018/15/20307-0514

DOI: $10.1097 / \mathrm{NMD} .0000000000000321$ attempt suicide. Therefore, the present qualitative study focuses on typical Plans (as explained below), motives, and the underlying selfregulatory processes of suicide attempters compared with nonsuicidal depressive individuals.

\section{Self-Regulatory Processes and Suicide Risk}

A self-regulatory process of behavior is a negative feedback loop (Carver and Scheier, 1998). This feedback loop includes personal goals and standards, which serve as reference values. The aim of selfregulatory behavior is to achieve these goals and standards and to reduce discrepancies between the actual state and the reference values. Previous research has investigated the relationship between goal engagement and psychological well-being. Brandtstadter and Renner (1990) found a relationship between the pursuit of goals and life satisfaction, less negative affect, depression, and neuroticism. Negative affect arises when goals are set very high and become unattainable (Emmons, 1992). In such a case, it would be advisable to relinquish those goals and to reengage in more attainable goals.

If goals can neither be achieved nor adapted, feelings of failure arise. These feelings may be related to suicide risk. O'Connor et al. (2012) found that difficulties in disengaging from goals and reengaging in new goals were predictors of rehospitalization after self-harm 2 years after an initial suicide attempt. Baumeister (1990) noted that suicidal behavior can be seen as a form of escape when a person falls far below his/her standards and wants to flee from this aversive state of negative affect. Although these findings are promising and provide valuable information about goal regulation processes in suicide attempters, there exists no study investigating prototypical goals and standards of suicide attempters and prototypical problems in self-regulated functioning. The aim of this article is to present a prototypical outline of self-regulated, goal-directed behavior and its underlying motives in suicide attempters. Furthermore, we were interested in examining the possibility of differences between individuals with a suicide attempt and depressive individuals without a suicide attempt regarding their Plans, motives, and self-regulated processes.

To investigate the Plans and motives of these two clinical groups, we used Plan Analysis, a method developed by Grawe and Caspar (Caspar, 2007a; Grawe, 1980). In its practical application, Plan Analysis is used primarily to develop psychotherapeutic case conceptualizations and individually tailored therapeutic strategies. In Plan Analysis, the patient's functioning is considered from an instrumental perspective. Based on the notion that behaviors tend to outlast and be elaborated if they have an obvious or more hidden conscious or unconscious advantage for an individual, hypothetical purposes of interpersonal as well as intrapsychic behavior are looked for (Caspar, 1995). This bottom-up search for purposes is complemented by the top-down question regarding means or strategies that an individual has developed for satisfying important motives or needs. Originating from the Plan concept by Miller et al. (1960), developed to overcome the limitations of traditional behaviorism, Plans are the basic unit also in clinical Plan Analysis. Plans consist of a purpose and the subordinate means serving the purpose. To highlight the difference to the everyday meaning of plans (above all that they are not necessarily conscious), Miller et al. (1960) 
have suggested writing Plan in the upper case. Plan Analysis is a wellelaborated concept used since the late 1970s for developing case conceptualizations as a basis for psychotherapy practice as well as for research (Berthoud et al., 2013; Caspar and Tuschen, 1987; Kramer et al., 2009).

Suicidal patients frequently have problems to talk frankly about their fears, thoughts, and worries. This often makes it difficult to understand the underlying causes and motives of a behavior and to recognize the important needs of those patients. In Plan Analysis, all available information of and about a patient (verbal and nonverbal behaviors, thoughts, beliefs, emotions, information from other sources such as questionnaires) can be used to infer a broad spectrum of Plans including conscious, explicit motives or goals as well as nonconscious, implicit motives or goals and strategies. This includes interpersonal (e.g., "reject the help of others") as well as intrapsychic (e.g., "avoid painful thoughts and emotions") strategies, which are often interwoven. Based on Plan Analysis, this study can therefore complement existing studies that focused on conscious aspects of goal-regulation in suicide attempters (e.g., O'Connor et al., 2012).

Plans are organized hierarchically and can be drawn twodimensionally in a Plan structure with means in a lower position and purposes or motives in a higher position. Observable behavior is depicted at the bottom of a Plan structure. Motives on the top of the hierarchy are largely equivalent to general human needs. In between are mediating Plans, which can be seen from a bottom-up or a top-down view; a Plan can serve as a mean or a Subplan to achieve a superordinate Plan (e.g., "resist developing new hopes" serves "avoid engaging in new attempts"). In the top-down view, the same Plan guides subordinate Plans and determines the goal component of the latter (e.g., "resist developing new hopes" guides the mean "maintain depression"). In a Plan structure, at least in healthy-functioning persons, important Plans are typically served by several subordinate Plans, yielding effectiveness and flexibility. Behaviors and Subplans are often constructed in such a way that they serve several guiding Plans at the same time (e.g., "end your life" serves "avoid being a burden on others" and at the same time "avoid being overwhelmed"). For this reason, a typical Plan structure shows upward and downward ramifications.

Although each person is different and Plan structures vary among patients even with the same diagnosis, researchers have found similarities of patients with a specific diagnosis or clinical problem. These similarities can be mapped onto a prototypical Plan structure (Caspar, 2007a). A prototypical Plan structure summarizes typical Plans of this group of patients and gives the therapist an overall idea of the ways in which many of these individuals function. A therapist trying to understand a patient belonging to a particular diagnostic or other group need not begin from scratch but can use parts of a prototypical structure as hypotheses for how this patient may function. This can be especially helpful for working with challenging individuals with a multifactorial disorder and complex problems like suicidal behavior. Prototypical Plan structures already exist for the following disorders: anxiety disorders (Caspar and Tuschen, 1987), depression (Caspar, 1995), borderline personality disorder (Berthoud et al., 2013), pedophilia (Drapeau et al., 2003), bipolar affective disorder (Kramer et al., 2009), and psychosomatic disorder (Caspar, 1995).

\section{METHODS}

\section{Sample}

Our sample of suicide attempters consists of 17 participants drawn from a larger study evaluating the Attempted Suicide Short Intervention Program (ASSIP) (Gysin-Maillart and Michel, 2013). These participants were hospitalized after a suicide attempt at the University Hospital of Psychiatry in Bern, Switzerland, and were included in the intervention program. It consists of four sessions followed by regular letters over 2 years. On an average of 4 weeks after admission, a narrative interview was conducted in the first session of the program. Based on information from the narrative interview, safety strategies were worked out within the following sessions of the ASSIP program to prevent future suicide attempts.

For our study purposes, we used the narrative interviews of the first session from the ASSIP study, which took about 1 hour. All patients provided informed consent that allows us to use their interviews for our study. Exclusions were limited to participants with language problems, unfit for an interview (actively psychotic), or taken into custody after admission. Ten patients were female (58.8\%). The mean (SD) age of this group was 38.9 (13.9) years (range, 20-62 years). ICD-10 (World Health Organization, 1993) diagnoses were available for 16 participants $(94.1 \%)$ and were drawn from the medical record during their emergency department visit. Nine $(52.9 \%)$ participants presented with major depression. Five (29.4\%) fulfilled criteria for adjustment disorder with a depressive reaction. One (5.8\%) met criteria for acute stress reaction, and another one (5.8\%), for posttraumatic stress disorder. Thirteen participants $(76.5 \%)$ reported no previous suicide attempt; three participants $(17.6 \%)$ had one previous suicide attempt, and one $(5.9 \%) \mathrm{re}-$ ported two previous suicide attempts (mean [SD], 0.29 [0.588]).

The group of nonsuicidal depressive patients consists of 17 participants taken from a larger study investigating motive-oriented therapeutic relationship (MOTHER; Caspar, 2007b). These participants were patients of the Psychotherapy Outpatient Clinic of the Department of Clinical Psychology and Psychotherapy at the University of Bern, Switzerland; they also gave informed consent, which allowed us to use their data and session videotapes for research purposes. Inclusion criteria of our study was a Diagnostic and Statistical Manual of Mental Disorders, 4th Edition (DSM-IV) (American Psychiatric Association, 2000) diagnosis of major depression. The diagnoses were established by staff trained in the use of the Structured Clinical Interview for DSM-IV (First et al., 2004). Exclusion criteria for this clinical group were a suicide attempt or acute suicidal thoughts. Ten patients were female (58.8\%). The mean (SD) age of this group was 37.1 (12.1) years (range, 22-64 years).

\section{Instruments}

Plan Analysis (Caspar, 2007a) was applied to both clinical groups. For the group of suicide attempters, we viewed the narrative interviews on videotape, which were conducted by the research group of the ASSIP study. In these interviews, participants described the processes of suicidal ideation development and how it came to a suicide attempt. It typically started with a clinician's opening intervention: "I would like you to tell me the story of what led to the suicidal crisis. Just let me listen to you." In the narrative approach, the clinician considers the patient as the agent of his/her own actions with a narrative competence to explain the subjective logic behind a suicide attempt. Thus, a joint understanding of the patient's suicidal crisis can be acquired, which is an important condition for establishing a collaborative working relationship with the suicidal patient (Gysin-Maillart and Michel, 2013). On the basis of information from the narrative interview, two trained raters inferred Plan structures for the group of suicide attempters.

The Plan structures of the nonsuicidal depressive patients were developed by nine trained master students. For developing the Plan structures, they viewed the intake interview and the first two psychotherapy sessions on videotape, which were recorded at the Psychotherapy Outpatient Clinic in Bern. The intake interview was a structured interview, in which the patient described his/her problems and reasons for therapy. Before developing the Plan structures of the current study, all raters had an extensive rater training over several weeks. During this training, they learned the method of Plan Analysis and developed Plan structures of several patients who were not part of the study sample. 
Differences between raters were discussed and finalized by consensus. Furthermore, all raters were blind to research questions. Analyses of interrater agreement for the Plan structures of suicide attempters were completed for a randomly chosen $10 \%$ of the cases $(2 / 17)$. For the group of depressive patients, we used the results of the interrater analyses of the total study sample by Caspar and Schmutz (2015). In this study, interrater analyses were completed for a randomly chosen $11 \%$ of the cases $(18 / 162)$.

\section{Procedure}

A prototypical Plan structure was established for each clinical group following a four-step procedure designed by Kramer et al. (2009) and Berthoud et al. (2013). These four steps were applied separately for each group:

1. Plans of the Plan structures from all patients of one clinical group were listed in an inventory. In the group of suicide attempters versus depressive patients, a total number of 632 versus 784 Plans were defined.

2. Semantically similar Plans were then grouped together into a Plan category, which can be seen as an aggregated Plan. For example, in the group of suicide attempters, semantically similar Plans like "break off your relationship and withdraw" in Plan structure 7 and "break off contact to your parents" in Plan structure 11 were aggregated to the Plan category "break off contact to important confidants and withdraw." Altogether, in the group of suicide attempters, 78 aggregated Plans were found, from 466 (74\%) of the 632 Plans. In the group of depressive patients, 76 aggregated Plans were found from $485(62 \%)$ of the 784 Plans.

3. Only the Plan categories with at least 7 Plans (40\%) of 17 Plan structures were transferred into the prototypical Plan structure. For example, if at least 7 semantically similar Plans (see below) were represented in the Plan category "break off contact and withdraw," this Plan category was transferred into the prototypical Plan structure. This threshold is higher compared with that in previous studies (Berthoud et al., 2013; Kramer et al., 2009) and was chosen so that the Plan structure of the subsample provides specific information of that clinical group.

4. Next, the prototypical Plans were examined based on their instrumental links. The same threshold of the prototypical Plan categories was applied to them. Finally, we composed an overall prototypical Plan structure for both clinical groups.

\section{RESULTS}

\section{Reliability of Plan Analysis}

For determining the interrater agreement of Plan Analysis, several methods have been developed depending on research questions and resources. In our study, we used the method of pair-wise comparison of all Plans. Therefore, we compared all Plans of two Plan structures (from rater A and B) from the same patient regarding three criteria. The first criterion is the equivalence of two Plans regarding their labeling (L). Equivalent Plans in two Plan structures are the basis for a comparison regarding the two additional criteria: superordinate Plans (SUP) and subordinate Plans (SUB). These criteria describe how equivalent Plans are embedded hierarchically in the Plan structures of raters A and B. A total average value of the interrater agreement is calculated with the values of the three categories. In previous studies, the pairwise comparison was limited to a certain number of selected Plans (e.g., 10 Plans; Berthoud et al., 2013; Kramer et al., 2009). This method is less conservative than the method used in this study, in which the interrater agreement of all Plans is included. The interrater reliability in the group of suicide attempters was $51 \%$, which is lower than in studies with a less conservative estimation but is still considered sufficient
(Benkert, 1997; Caspar, 2007a). The interrater analysis in the depressive patient sample yielded an overall average of $59 \%$.

\section{Prototypical Plan Structures}

The prototypical Plan structures of the two clinical groups are shown in Figures 1 and 2. In the group of suicide attempters, 29 Plans could be transferred into the prototypical Plan structure. In the group of depressive patients, 33 Plans could be transferred into the prototypical Plan structure. We found 11 prototypical Plans that occur in the prototypical Plan structures of both clinical groups (Plans printed in bold shown in Figs. 1 and 2). Furthermore, we depicted those instrumental links between prototypical Plans in the Plan structures that became prototypical by passing the defined threshold of $40 \%$. With some Plans, we depicted the instrumental links by a dashed line, meaning they did not become prototypical in our sample but were close to the threshold $(30 \%-39 \%)$ but show the potential direction of instrumentality.

In the following section, we describe the main characteristics of the suicide attempters' prototypical Plan structure with respect to overlapping Plans compared with the group of nonsuicidal depressive patients. The numbers in parentheses after the Plan designation that exist in both clinical groups represent the occurrence of the Plan in the group of suicide attempters versus nonsuicidal depressive patients. Beginning at the top of the Plan structure, the need "protect your selfesteem" (16 vs. 12) seems to be very important in both groups. They both indicate overlapping avoidance Plans to protect their self-esteem, for example, "avoid being rejected" (13 vs. 9) and "avoid being criticized" (12 vs. 8).

However, suicide attempters and depressive patients also differ in their strategies to satisfy their need of self-esteem protection. In the Plan structure of suicide attempters, the Plans "break off contact to important confidants and withdraw" (11) and "end your life" (15) serve multiple avoidance Plans like "avoid being disappointed and harmed" (9), "avoid to fail" (10), "avoid appearing as weak" (8), and "avoid being a burden on others" (10). In addition, they show the Plan "try to cope with your problems by yourself" (8) with connections to the interpersonal avoidance Plans "avoid being a burden on others" and "avoid appearing as weak."

In contrast, depressive patients seek to adapt to persons and situations ("adapt yourself" [9]) and show the Plan "devalue yourself" (7), which both serve to "anticipate critique" (8) as specific strategies of this group serving the protection of their self-esteem. Other Plans related to self-esteem protection in this group are "avoid assuming responsibility" (7) and "maintain self-determination" (9). With respect to approach Plans, we found the Plan "perform perfectly on the job" ( 9 vs. 7) in both groups, which serves the Plan "search for acknowledgement" (7vs. 13) as further means to protect self-esteem. Serving this same Plan, the Subplan "meet high demands and expectations" (9) has been found additionally in suicide attempters.

The need for control is reflected by the Plan "maintain emotional control" (12) in suicide attempters and "avoid losing control" (15) in depressive patients. Both groups try to "avoid painful thoughts and emotions" (9vs. 9) to maintain an emotionally stable state. The Plan "avoid dealing with your problems" (9vs. 7) can also be found in both groups as a strategy to maintain control over one's life. This simultaneously serves the Plan "avoid appearing as weak" in the group of suicide attempters. In this group, certain emotion-regulating Plans were also identified, such as "avoid being overwhelmed" (14), "avoid repeated negative states and experiences" (11), and "reduce tension" (8) to maintain emotional control and to "stop mental pain" (11). In comparison, some depressive patients use the Plan "avoid being alone" (7) for maintaining control. Moreover, they use interpersonal control strategies like "keep interpersonal distance" (9) and "avoid opening yourself" (8) to avoid losing control over their lives. The latter Plan is also applied by suicide attempters (12), but in that group, it serves the 


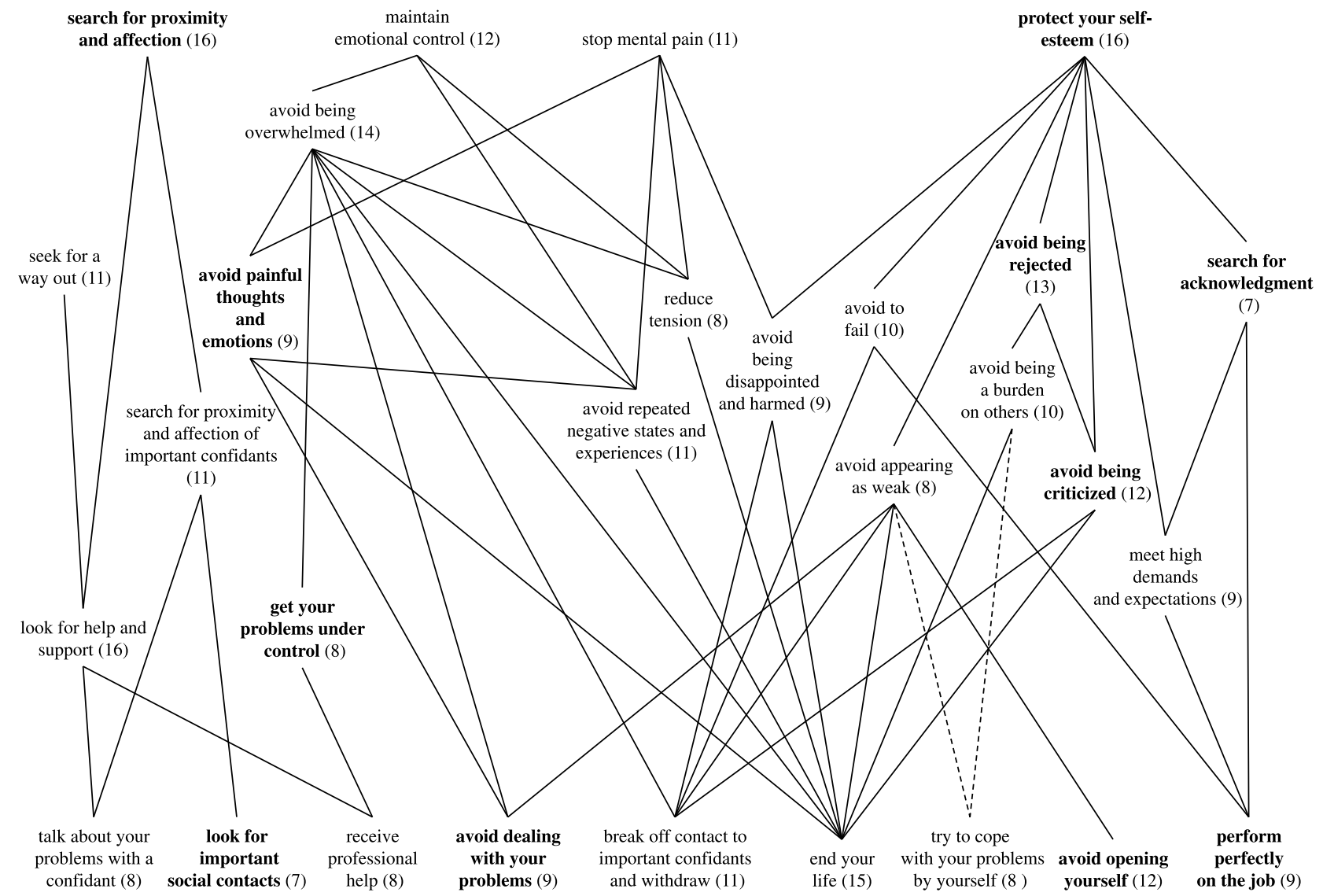

FIGURE 1. Prototypical Plan structure of suicide attempters. The numbers in brackets represent the occurrence of each Plan in the 17 Plan structures. Plans in bold occur in the prototypical Plan structures of both clinical groups. Instrumental links between two Plans are depicted by smooth lines. Links that became not prototypical are marked with a dashed line.

Plan "avoid appearing as weak" to ensure self-esteem protection instead of maintaining control. Although both groups try to avoid addressing problems, they nonetheless show the Plan "get your problems under control" (8vs. 12) to maintain control over their lives. For depressive patients, this Plan also serves the high-level Plan "enjoy your life" (9) next to other strategies like "take care of yourself" (12) and "work hard on yourself/change something" (9), which can be found only in the Plan structure of this group.

In both clinical samples, the need for affiliation is represented by the approach Plan "search for proximity and affection" (16 vs. 17). In an attempt to satisfy this need, both groups use the Plan to "look for important social contacts" (7 vs. 9). Apart from these similarities, suicide attempters and depressive patients differ widely among their lower-level Plans serving to fulfill this need. Suicide attempters mainly show support-seeking Plans like "receive professional help" (8) and "look for help and support" (16) to "seek for a way out" (11), and to satisfy the need for proximity and affection. By contrast, depressive patients mostly use interpersonal control strategies like "show how bad you feel" (8) to "cause care" (7), "maintain the relationship with your partner" (7), and "please others" (9). Nevertheless, both groups also have overlapping Plans for realizing this need: "talk about your problems with a confidant" (8) on the part of suicide attempters and "open yourself" (9) on the part of depressive patients.

\section{DISCUSSION}

The purpose of this study was to investigate Plans, motives, and underlying self-regulated processes of suicide attempters compared with those of nonsuicidal depressive patients. First, we will discuss the characteristics of the two prototypical Plan structures with respect to current theoretical models for suicidal behavior. Then, we will address potential clinical implications of these findings regarding suicide prevention and interventions.

Regarding self-esteem protection, we found that Plans of suicide attempters reflect the high personal standards of these individuals and the belief that others (e.g., family members) hold high expectations of them. They fear failure, especially in the professional context, and generally do not want to be considered weak nor be criticized by others. These findings support the results of previous studies regarding the relationship between perfectionism and suicidality. O'Connor (2007) concluded that social perfectionism, the "belief that others hold unrealistically high expectations... and... would only be satisfied with these standards" (p. 699), is related to suicidality and could distinguish between suicide ideators, attempters, and controls in both clinical and population-based studies. Beevers and Miller (2004) found in a depressive inpatient sample that perfectionism directly predicted suicidal ideation 6 months after hospitalization, independent of hopelessness.

Nonsuicidal depressive patients in our sample also avoid being criticized by others, but they use different strategies to reach this goal. They devaluate themselves and defer their own needs ("adapt yourself") to anticipate negative reactions from others. This can be considered as an accommodative coping strategy. In contrast, suicide attempters withdraw from their social environment and show suicidal behavior to avoid being criticized.

The findings of a study conducted by Frank et al. (2007) about self-esteem of depressed patients can provide a possible explanation 


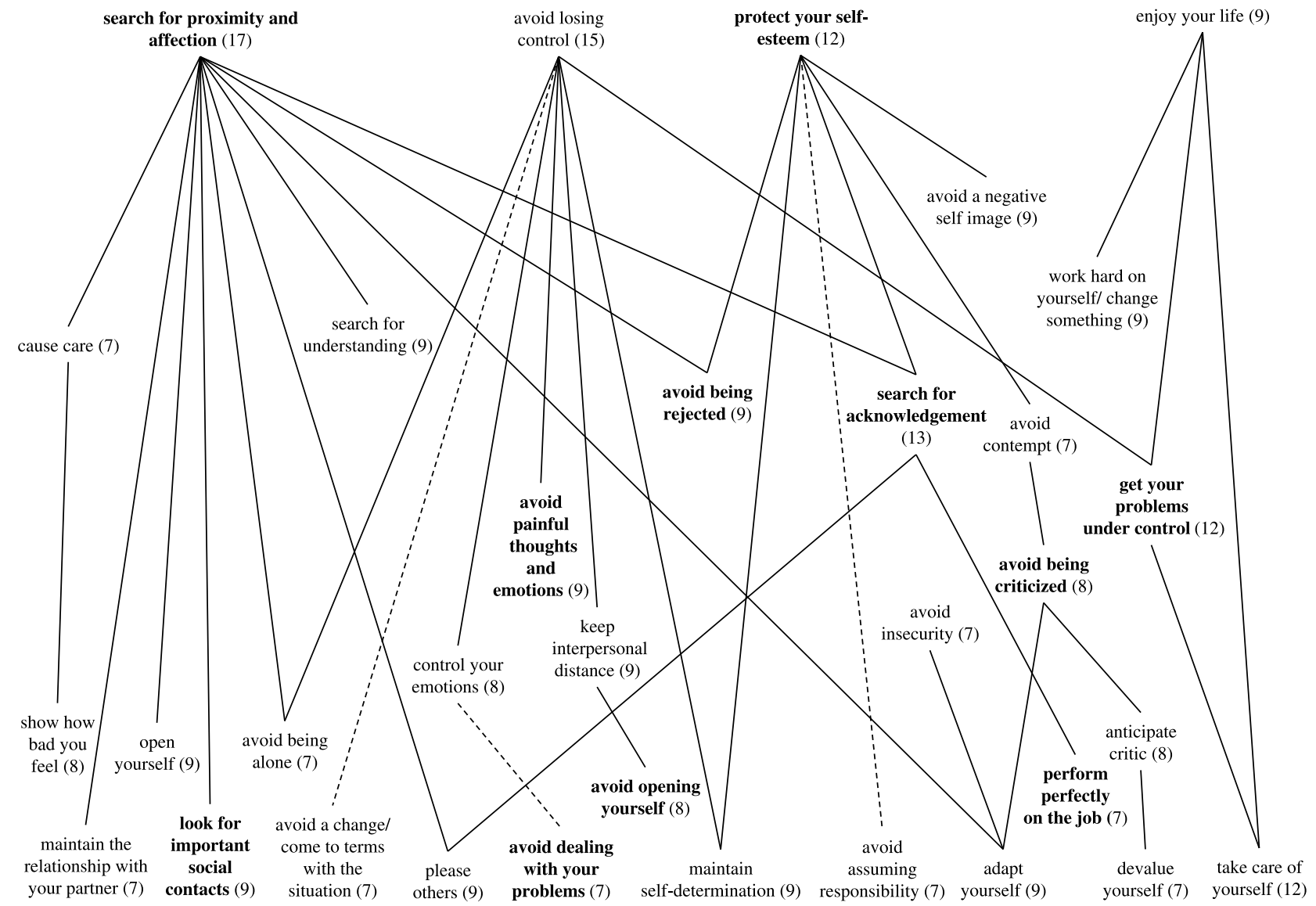

FIGURE 2. Prototypical Plan structure of nonsuicidal, depressive patients. The numbers in brackets represent the occurrence of each Plan in the 17 Plan structures. Plans in bold occur in the prototypical Plan structures of both clinical groups. Instrumental links between two Plans are depicted by smooth lines. Links that became not prototypical are marked with a dashed line.

for these differences. They found that depressed patients with and without suicidal ideation have significantly lower explicit self-esteem compared with nondepressed controls. However, the implicit self-esteem of depressed patients with suicidal thoughts was higher than in depressed patients without suicidal thoughts and did not differ compared with the control group. These findings might explain why nonsuicidal depressive patients in our study developed Plans of devaluing and adapting themselves. Low implicit self-esteem might be expressed by the fact that this group partially adjusted their internal goals and expectations of themselves to the perceived reality and gave up high expectations and striving for important goals. Prototypical Plans that we have found in this group, such as "avoid assuming responsibility" and "come to terms with the situation," could provide further evidence for this selfregulation process. The discrepancy between the implicit and explicit self-esteem in depressive patients with suicidal ideation might be a hint that these individuals neglected this accommodative process.

Baumeister (1990) concludes that suicidal individuals frequently experience a combination of unrealistically high expectations and recent failures and setbacks. The discrepancy between the implicit and explicit self-esteem in this group may reflect this experience. In this situation, feelings of being entrapped could arise. These feelings are related to the "cry of pain" hypothesis, a situation in which one feels entrapped when he/she is defeated and there is no possibility to escape, resulting in suicidal behavior as a response to this aversive state (Williams and Pollock, 2000). This is consistent with our findings of suicide attempters who felt desperate, overwhelmed, anxious, or restless when important Plans related to their self-esteem were blocked.
Although previous research has demonstrated that depressive patients also show feelings of entrapment (Taylor et al., 2011), the accommodative coping effort of depressive patients in our sample may have potentially reduced this feeling or prevented the development of such a strong escape-motivation found in suicide attempters. More research is needed to clarify whether the degree of entrapment and escape-motivation differ between suicide attempters and nonsuicidal depressive patients and the extent of the influence of goal self-regulation on these variables.

Furthermore, the prototypical Plan structure of suicide attempters indicates that suicidal behavior serves not only as an escape route for reducing inconsistency regarding self-esteem but also as a method to stop mental pain. Suicide attempters in our sample described mental pain as "heartache" or "pain in one's mind," which was perceived as unbearable and accompanied by a deep feeling of despair. The Plan "stop mental pain" can be associated with the concept of "psychache" proposed by Shneidman (1993), an acute state of psychological pain caused by thwarted essential needs. Shneidman postulates that when pain reaches a high intensity, a person commits suicide as an attempt to escape this suffering. This is in line with our results, which indicate a connection between the Plans "end your life" and "stop mental pain." The Plan structure of suicide attempters can provide detailed information of what causes mental pain beyond frustrated needs. Our results demonstrate that mental pain is caused primarily by defeat and rejection. Disappointments and the feeling of being harmed as well as repeated negative states and experiences were the main reasons for mental pain. In more detail, suicide attempters reported that negative states and experiences were depressive episodes, rape, job loss, or an 
unbearable physical condition. Disappointments were mainly reported in relation to close family members or were experienced at work. In addition, suicidal behavior serves to reduce tension with the motive "stop mental pain." This phenomenon is consistent with the assumption that mental pain is associated with a state of internal perturbation (Shneidman, 1999). Previous research demonstrated that mental pain is a unique contributor to the prediction of attempter status and the number of lifetime attempts when depression and hopelessness are also included (Troister and Holden, 2010). This is in accordance with our finding that the Plan "stop mental pain" is located on the highest level of the Plan structure and cannot be further described by any other superior goal or basic need.

Comparing the two Plan structures reveals important differences between suicide attempters and nonsuicidal depressive patients in terms of dealing with problems and seeking support. Although both groups avoid dealing with their problems, in the Plan structure of suicide attempters, several problem-solving (e.g., "receive professional help") and support-seeking (e.g., "talk about your problems with a confidant," "look for important social contacts") strategies are presented. However, it is important to mention that in most suicide attempters, problemsolving and support-seeking Plans were deactivated during the suicidal crisis or were established only after the suicide attempt. Most suicide attempters in our sample reported having social support but avoided using them as their condition worsened. This fostered dysfunctional processes of problem-solving and emotion regulation.

A possible reason for the lack of support-seeking strategies during stressful life situations in suicide attempters can be provided by the avoidance Plans "avoid being a burden on others" and "avoid to fail." This is related to the construct "perceived burdensomeness" of the "interpersonal theory of suicide" by Joiner et al. (2009). This construct comprises two dimensions: the person perceives him/herself as a burden to others on the one hand and as a failure on the other hand. This promotes feelings of self-hate and shame. Whereas the Plan "avoid being a burden on others" may be related to the first dimension, the Plan "avoid to fail" may be associated with the second dimension. As one can see from the instrumental links in the Plan structure of suicide attempters, these Plans may not only be a cause for not seeking support ("try to cope with your problems by yourself") but also a motivation to develop a suicidal desire ("end your life"). This is in line with the interpersonal theory stating that perceived burdensomeness is a sufficient cause for developing suicidal desire.

In comparison, depressive patients apply several interpersonal control strategies, such as "show how bad you feel," "maintain the relationship with your partner," "please others," and "avoid being alone" to satisfy their need for affiliation. Simultaneously, these interpersonal control strategies often lead to significant others taking special care and protective monitoring. This can serve as a protective factor for not developing suicidal behavior. The "avoid being alone"-"avoid losing control" path specifically demonstrates that depressive patients use others to maintain control. This may be a lifesaving relationship, at least as long as this Plan is not thwarted (i.e., if the Plan "avoid being alone" is thwarted, the person cannot live in line with this Plan and thus feels isolated) and the individual receives protective treatment by others. Moreover, we have identified several approach Plans (e.g., "take care of yourself," "enjoy your life") in depressive patients, which may serve as additional self-protecting factors.

Our findings suggest several clinical implications. First, it is important to know that suicide attempters visited a general practitioner more frequently before a completed suicide or suicide attempt, suggesting that the reasons for the visits to health professionals are related to the development of a suicidal crisis (Appleby et al., 1996; Michel et al., 1997). The prototypical Plan structure of suicide attempters can thus be a tool for clinicians to detect critical Plans in the statements and behavior of individuals who are potentially at high risk for a suicide attempt. For instance, if a patient perceives himself/herself as a burden to others, has the sense of having failed, is afraid of the recurrence of negative states, experiences disappointments, or is in a condition which she/he perceives as unbearable coupled with mental pain, this could be an indication of an increased risk for suicide. However, it is still a challenging task for clinicians to predict who will attempt suicide and who will not. We want to address this critical issue by pointing to several Plans of the suicide attempters' Plan Structure that seem particularly important to us in developing suicidal behavior. On the one hand, there are Plans representing more stable characteristics of the person, for example, Plans closely linked to perfectionism (e.g., "meet high demands and expectations," "avoid to fail"). These Plans might represent a person's vulnerability for developing suicidal behavior. If these Plans are threatened and the individual is hindered from fulfilling his/her perfectionistic demands, more state-dependent risk processes are triggered. Because of the threat of important Plans, suicide attempters of our sample often experienced a feeling of high internal tension, frequently accompanied by insomnia. This state was supplemented by extreme feelings of being overwhelmed resulting from subjectively exhausting life conditions. Then, suicidal behavior was often used as a strategy to decrease high tension and to escape from being overwhelmed (see the "end your life"-"reduce tension"-“avoid being overwhelmed" path). Therefore, insomnia and physiological hyperarousal expressed as agitation and feelings of high internal tension should be routinely considered for suicide risk evaluation. A further frequently observed process in our suicidal patients was the extreme withdrawal from social environment shortly before their suicide attempt. This behavior was, in turn, largely triggered by Plans related to perfectionisms and self-esteem protection. Social withdrawal represents a perilous risk process for exceeding the threshold to suicidal behavior. On the one hand, it fosters a cognitive and emotional narrowing, in which positive future-oriented thoughts are hardly accessible (Rasmussen et al., 2010). On the other hand, through social withdrawal, the person (consciously or non-consciously) creates "favorable" conditions to be able to attempt suicide uninterruptedly. Social withdrawal should therefore be closely monitored.

Overall, the prototypical Plan structure of suicide attempters includes important warning signs of a suicidal crisis, which are no longer to be considered in isolation from one another but are instrumentally linked and thus point to a number of potential interventions for reducing suicide risk. For example, suicidal behavior as a dysfunctional solution for avoiding to be criticized, harmed, and disappointed could indicate implementing a training of social problem-solving in therapy with suicide attempters to reduce suicide risk.

As a further clinical implication, the prototypical Plan structure can help to create favorable conditions for a strong therapeutic alliance, which is sometimes a special challenge for clinicians working with suicidal patients in times of managed care and time pressures. An essential element of a beneficial therapeutic alliance is what is designated a MOTHER (Caspar, 2007b). For this purpose, the prototypical Plan structure of suicide attempters can be used as a compass to identify important patient motives and meet them through appropriate therapeutic behavior. For example, facing a suicidal patient with the "break off contact" Plan activated to "avoid appearing as weak," the clinician will have a supportive and empathetic nonverbal attitude and will assure the patient that the clinician understands his/her desperate situation and does accept him/her as a valuable person independently of his/her problems. This attitude may help the patient to talk about his/her problems and may reduce the patient's sense that everything is at stake when she/he appears weak. The result should be that it is no longer necessary for the patient to use his/her problematic behavior, for example, withdrawal, suicidal behavior, if these goals are satisfied within the therapeutic relationship. Furthermore, motive-orientedness by considering the Plans "avoid being disappointed and harmed" and "avoid repeated negative states and experiences" means to convey to the patient that the clinical setting is a secure place, in which he/she can rely on the clinician without having the sense of being a burden. It is an alarming fact 
that $38 \%$ of suicide attempters who do attend treatment drop out from outpatient treatment after 3 months and $73 \%$ are not in treatment after 1 year (Lizardi and Stanley, 2010). Therefore, knowing such critical and frequently occurring Plans in suicide attempters can help the clinician to establish a reliable therapeutic alliance faster, which might be lifesaving. Evidence for the effectiveness of MOTHER can be provided by a pilot study conducted by Kramer et al. (2011), in which a reduction of interpersonal problems and a stronger therapeutic alliance was found in the early-phase treatment of patients with borderline personality disorder. In a recent study by Kramer et al. (2014), these findings could be replicated and extended in a larger ten-session randomized controlled trial (RCT).

The present study also has a number of limitations that might have influenced some of the results. One limitation is the small number of included patients and the limited homogeneity of diagnoses in suicide attempters, meaning that findings are only preliminary results. However, in most RCT studies with suicide attempters like the ASSIP study, the samples are heterogeneous because of low recruitment rate of suicide attempters (Brown et al., 2005; Slee et al., 2008). Nevertheless, $83 \%$ of our sample of suicide attempters were affected by a depressive mood, which might make our sample less heterogeneous. With regard to the validity of diagnoses, suicide attempters' diagnoses were drawn from medical records as opposed to conducting structured clinical interviews in the group of depressive patients. As a consequence, the diagnoses of suicide attempters might be less valid compared with the diagnoses of depressive patients. However, 13 of the 17 suicide attempters in our sample have had at least one outpatient treatment before or during current hospitalization, where an experienced psychiatrist or psychologist established a valid diagnosis. During the diagnostic procedure at the psychiatric ward, the psychiatrist on duty carefully considered this diagnosis and checked whether it could be confirmed or had to be adapted. Moreover, most of the suicide attempters stayed on the psychiatric ward for several weeks, during which the initial diagnosis at hospitalization was checked again by the treating psychiatrist. This fact might weaken the limitation of different diagnostic procedures.

Moreover, different material was used to develop the Plan structures of the two groups. The videotaped material of the depressive patients was more extensive, which could mean that we have missed some information in the group of suicide attempters. Nevertheless, the number of inferred Plans in the Plan structures of the two samples did not differ substantially from each other. Furthermore, the interviews of the two clinical groups were both problem centered, which might reduce the influence of different material.

A further limitation is the retrospective nature of the narrative interview in the group of suicide attempters. Conducting the interview not immediately after the suicide attempt might lead to a possible recall bias. However, several aspects (e.g., surgery after a suicide attempt, longer reflection of patients regarding study participation) played a role for a delay of the interview, which were sometimes difficult to overcome.

Another limitation refers to the process of developing Plan structures. The inferring of Plans can never be fully objective, so we cannot completely rule out a possible rater bias. Yet, to minimize this impact, the raters were blind to the research questions, design, and goal of the study. Moreover, we followed a number of recommendations for the use of Plan Analysis as a research method to prevent a systematic bias (Caspar, 1995). For example, extensions are recommended as an intermediate step to enhance the behavior-to-Plan inference process. An extension is a rater's transcript containing interpretations of raw video material to question interpretations at any time.

\section{CONCLUSIONS}

Suicidal behavior is a complex phenomenon, and predicting suicide risk is still rather limited. If we want to further understand the causes of suicide, we need to move beyond the evaluation of psychiatric risk factors, and one way may be the investigation of underlying motives and internal processes of suicidal individuals. Our findings suggest that suicidal behavior is closely linked to high standards and expectations and can be seen as a form of escape to protect their selfesteem and to avoid being a burden on others. The motives behind the suicide attempt and even the attempt itself are often related to feelings of failure, shame, and self-blame. Consequently, suicide attempters withdraw from their social environment and deny seeking available help. For that reason, it is important for clinicians to understand the motives and inner logic of the suicide attempt, which may help to engage the patient in a therapeutic relationship and prevent future suicide attempts.

\section{DISCLOSURES}

The authors declare no conflict of interest.

\section{REFERENCES}

American Psychiatric Association (Ed) (2000) Diagnostic and statistical manual of mental disorders (4th ed). Washington, DC: APA.

Appleby L, Amos T, Doyle U, Tommenson B, Woodman M (1996) General practitioners and young suicides. Br J Psychiatry. 168:330-333.

Baumeister RF (1990) Suicide as escape from self. Psychol Rev. 97:90-113.

Beevers CG, Miller IW (2004) Perfectionism, cognitive bias, and hopelessness as prospective predictors of suicidal ideation. Suicide Life Threat Behav. 34:126-137.

Benkert F (1997) Reliability testing for plan analyses. Unpublished master's thesis Tübingen, Germany: Eberhard Karls University.

Berthoud L, Kramer U, Roten Y, Despland JN, Caspar F (2013) Using plan analysis in psychotherapeutic case formulation of borderline personality disorder. Clin Psychol Psychother. 20:1-9.

Bertolote JM, Fleischmann A (2002) Suicide and psychiatric diagnosis: A worldwide perspective. World Psychiatry. 1:181-185.

Blair-West GW, Cantor CH, Mellsop GW, Eyeson-Annan ML (1999) Lifetime suicide risk in major depression: Sex and age determinants. J Affect Disord. 55:171-178.

Brandtstadter J, Renner G (1990) Tenacious goal pursuit and flexible goal adjustment: Explication and age-related analysis of assimilative and accommodative strategies of coping. Psychol Aging. 5:58-67.

Brown GK, Have TT, Henriques GR, Xie SX, Hollander JE, Beck AT (2005) Cognitive therapy for the prevention of suicide attempts: A randomized controlled trial. J Am Med Assoc. 294:563-570.

Carver CS, Scheier MF (Eds) (1998) On the self-regulation of behavior. New York: Cambridge University Press.

Caspar F (Ed) (1995) Plan analysis. Towards optimizing psychotherapy. Seattle, WA: Hogrefe.

Caspar F (Ed) (2007a) Beziehungen und Probleme verstehen: Eine Einführung in die psychotherapeutische Plan Analyse [Understanding relationships and problems: An introduction to the psychotherapeutic plan analysis]. Bern, Switzerland: Huber.

Caspar F (2007b) Plan analysis. In Eells TD, ed. Handbook of psychotherapy case for mulations (2nd ed, pp 221-289). New York: Guilford Press.

Caspar F, Schmutz I (2015) Complementary therapeutic relationships: Conditions and relation to therapy success. Manuscript in preparation.

Caspar F, Tuschen B (1987) Die Angst und ihre Umgebung. Eine Stellungsnahme zur kognitiven Therapie von Angst. [Anxiety and its surroundings. An elaboration on cognitive therapy of anxiety]. Hypnose und Kognition. 4:64-82.

Drapeau M, Körner A, Brunet L, Granger L, de Roten Y, Caspar F (2003) L'analyse des mobiles comme méthode qualitative: Un exemple tiré d'une recherche sur le traite- ment des agresseurs sexuels pédophiles [Plan analysis as qualitative method: An example from a research on the treatment of child molesters] Recherches Qualitatives. 23:27-46.

Emmons RA (1992) Abstract versus concrete goals: Personal striving level, physical illness, and psychological well-being. J Pers Soc Psychol. 62:292-300. 
First MB, Spitzer RL, Williams JB, Gibbons M (Eds) (2004) Structured clinical interview for DSM-IV. New York: Biometrics Research Department.

Frank E, De Raedt R, Dereu M, Abbeele V (2007) Implicit and explicit self-esteem in currently depressed individuals with and without suicidal ideation. J Behav Ther Exp Psychiatry. 38:75-85.

Grawe K (1980) Die diagnostisch-therapeutische Funktion der Gruppeninteraktion in verhaltenstherapeutischen Gruppen. In Grawe K (Ed), Verhaltenstherapie in Gruppen [Behavioral therapy in groups] (pp 88-223). München, Germany: Urban \& Schwarzenberg.

Gysin-Maillart A, Michel K (Eds) (2013) Kurztherapie nach Suizidversuch. ASSIP - Attempted Suicide Short Intervention Program. Therapiemanual. Bern, Switzerland: Huber.

Joiner TE, Van Orden KA, Witte TK, Rudd MD (Eds) (2009) The interpersonal theory of suicide: Guidance for working with suicidal clients. Washington, DC: American Psychological Association.

Kramer U, Berger T, Caspar F (2009) Psychotherapeutic case conceptualization using plan analysis for bipolar affective disorder. J Clin Psychol. 65:352-367.

Kramer U, Berger T, Kolly S, Marquet P, Preisig M, de Roten Y, Caspar F (2011) Effects of motive-oriented therapeutic relationship in early-phase treatment of borderline personality disorder. A pilot study of a randomized trial. J Nerv Ment Dis. 199:244-250.

Kramer U, Kolly S, Berthoud L, Keller S, Preisig M, Caspar F, Despland J-N (2014) Effects of motive-oriented therapeutic relationship in a ten-session general psychiatric treatment of borderline personality disorder: A randomized controlled trial. Psychother Psychosom. 83:176-186.

Linehan MM (2008) Suicide intervention research: A field in desperate need of development. Suicide Life Threat Behav. 38:483-485.

Lizardi D, Stanley B (2010) Treatment engagement: A neglected aspect in the psychiatric care of suicidal patients. Psychiatr Serv. 61:183-1191.

Michel K, Runeson B, Valach L, Wasserman D (1997) Contacts of suicide attempters with GPs prior to the event; a comparison between Stockholm and Bern. Acta Psychiatr Scand. 95:94-99.

Miller GA, Galanter E, Pribram KH (Eds) (1960) Plans and the structure of behavior. New York: Holt.
Nock MK, Hwang I, Sampson NA, Kessler RC (2010) Mental disorders, comorbidity and suicidal behavior: Results from the National Comorbidity Survey replication. Mol Psychiatry. 15:868-876.

O'Connor RC (2007) The relations between perfectionism and suicidality: A systematic review. Suicide Life Threat Behav. 37:698-714.

O'Connor RC, O'Carroll RE, Ryan C, Smyth R (2012) Self-regulation of unattainable goals in suicide attempters: A two year prospective study. J Affect Disord. 142:248-255.

Powell J, Geddes J, Deeks J, Goldacre M, Hawton K (2000) Suicide in psychiatric hospital in-patients-Risk factors and their predictive power. $\mathrm{Br} J$ Psychiatry. 176:266-272.

Rasmussen S, Fraser L, Gotz M, MacHale S, Mackie R, Masterton G, McConachie S, O'Connor RC (2010) Elaborating the cry of pain model of suicidality: Testing a psychological model in a sample of first-time and repeat self-harm patients. Br J Clin Psychol. 49:15-30.

Shneidman ES (1993) Suicide as psychache. J Nerv Ment Dis. 181:145-147.

Shneidman ES (1999) Pertubation and lethality: A psychological approach to assessment and intervention. In Jacobs DG (Ed), The Harvard Medical School guide to suicide assessment and intervention (pp 83-97). San Francisco: Jossey-Bass.

Slee N, Garnefski N, van der Leeden R, Arensman E, Spinhoven P (2008) Cognitivebehavioural intervention for self-harm: Randomised controlled trial. Br J Psychiatry. 192:202-211.

Taylor PJ, Gooding P, Wood AM, Tarrier N (2011) The role of defeat and entrapment in depression, anxiety, and suicide. Psychol Bull. 137:391-420.

Troister T, Holden RR (2010) Comparing psychache, depression, and hopelessness in their associations with suicidality: A test of Shneidman's theory of suicide. Pers Individ Differ. 49:689-693.

Williams JMG, Pollock LR (2000) The psychology of suicidal behavior. In Hawton K, van Heeringen $\mathrm{K}$ (Eds), The international handbook of suicide and attempted suicide (pp 79-93). Chichester, England: John Wiley \& Sons.

World Health Organization (1993) The ICD-10 classification of mental and behavioral disorders: Diagnostic criteria for research. Geneva: World Health Organization.

World Health Organization (2008) Suicide statistics: Introduction. Retrieved March 24, 2013, from http://www.who.int/mental_health/prevention/suicide/suicideprevent/ en/print.html. 